\title{
Impact of Hardware Impairment Analysis on Channel Capacity Bounds of MIMO Antenna Configurations
}

\author{
Amjad Quazi, Sanjeev Kumar Gupta, Paresh Rawat
}

\begin{abstract}
: with the increasing demand $f$ the higher data reates the utilization of the MIMO system have exponentially increased in the cellular communication systems. There are many reasons due to which the performance of the MIMO systems degrades in real time. The major challenge is the any kind of noise or erro in the system due to hardware issues and problems. The any kind of hardware error is called as hardware impairment in the systems. These impairments are represented by Kapa values in the systems. Paper is primarily focused to define and evaluate the impact of the hardware impairment on the system performance of MIMO. The paper first defines the basic MIMO systems antenna configurations and then the channel capacity performance is compared with and without hardware impairments. the antenna configurations depends on the number of input and output antenna in the system. As the number of antennas increases the probability of having hardware impairment also increases. This may reduce the capacity performance significantly. New channel capacity formulation is given for antenna configurations. Paper evaluated the performance under the different channel sizes on the capacity. Finally the capacity of the channel is plotted as the function of the different Signal to noise ratios. It is concluded the MIMO system performance is degraded under the presence of the hardware impairments.
\end{abstract}

Keywords: About four key words or phrases in alphabetical order, separated by commas.

\section{INTRODUCTION}

M Multiple-Input Multiple-Output (MIMO) technology uses significantly increased in the $3 \mathrm{G}-4 \mathrm{G}$ of the cellular system. It is a wireless technology that uses multiple transmitters and receivers to transfer more data at the same time. MIMO technology primarily takes advantage of a radio-wave phenomenon called multipath where transmitted information bounces off walls, ceilings, and other objects, reaching the receiving antenna multiple times via different angles and at slightly different times. the journal, rectification

Revised Manuscript Received on February 05, 2020.

* Correspondence Author

Prof. Mohd Amzad*, Department of Electronics and Communication Engineering, SISTec College, RGPV University, Bhopal, India

Dr. Sanjeev Kumar Gupta, Department of Electronics and Communication Engineering, RNTU Technical University, Bhopal, India.

Dr. Paresh Rawat, Department of Electronics and Communication Engineering, SISTec College, RGPV University, Bhopal, India

(C) The Authors. Published by Blue Eyes Intelligence Engineering and Sciences Publication (BEIESP). This is an open access article under the CC BY-NC-ND license (http://creativecommons.org/licenses/by-nc-nd/4.0/) is not possible.

The MIMO innovation influences the multipath conduct by utilizing multiple, number of the "smart" transmitters and beneficiaries with an additional "spatial" measurement to significantly expand execution and range. MIMO permits multiple reception apparatuses to send and get multiple spatial streams simultaneously.

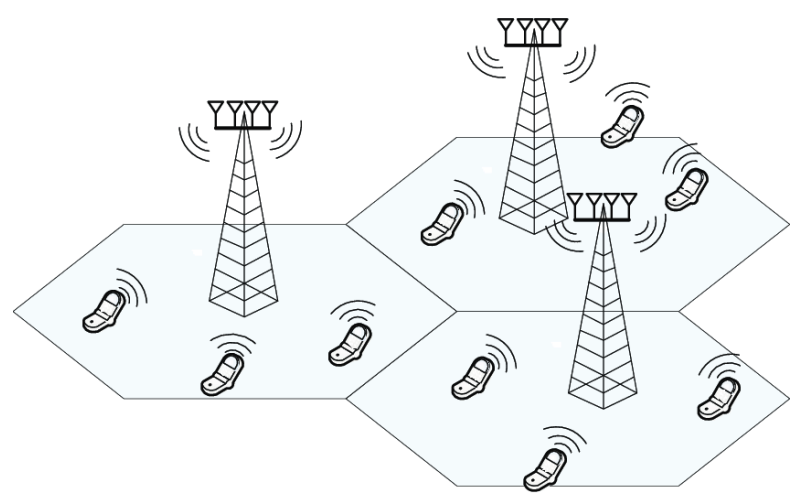

Figure 1 MIMO Technology for the cellular system

MIMO makes reception apparatuses work more brilliant by empowering them to consolidate information streams landing from various ways and at various occasions to viably build collector signal-catching force. Keen recieving wires utilize spatial decent variety innovation, which puts surplus radio wires to great use. In the event that there are a larger number of reception apparatuses than spatial streams, the extra radio wires can include recipient assorted variety and increment extend

MIMO correspondences channels give a fascinating answer for the multipath challenge by requiring multiple signal ways. In actuality, MIMO frameworks utilize a blend of multiple radio wires and multiple signal ways to pick up information on the interchanges channel. By utilizing the spatial element of a correspondences interface, MIMO frameworks can accomplish essentially higher information rates than customary single-input, single-output (SISO) channels. In a 2 x 2 MIMO framework, signals proliferate along multiple ways from the transmitter to the recipient reception apparatuses. Utilizing this channel information, a collector can recuperate autonomous streams from every one of the transmitter's radio wires. A 2 × 2 MIMO framework produces two spatial streams to viably twofold the greatest information pace of what may be accomplished in a conventional 1 x 1 SISO channel

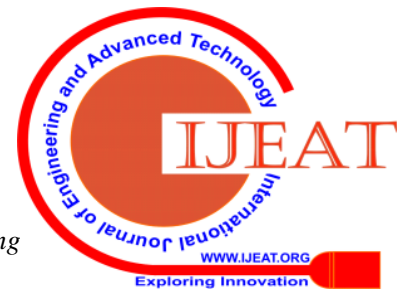




\section{Assumptions}

Some important assumptions are made:

- $\quad$ There is only a single user transmitting at any given time, so the received signal is corrupted by AWGN (Additive White Gaussian Noise) only.

- The communication is carried out in packets that are of shorter time span then the coherence time of the channel.

This means that the channel is constant during the transmission of a packet.

- The channel fading is frequency-flat. This means that the channel gain can be represented by a complex number. This also means that the transmission is very narrowband and the complex number, which represents the fading, is constant over the bandwidth.

\section{REVIEW OF WORK}

Y. Gizlenmistir et al., [1] Cellular correspondence networks are reliably creating to manage up the expanding enthusiasm for remote correspondence. This intrigue has been giving more repeat run, higher cell thickness, and higher otherworldly effectiveness. We base on improving energy and ghostly productivity. Upgrading these two parameters fulfills the difficulties of remote system correspondence. LTE Enormous MIMO is one of the contemporary thoughts promising mind boggling updates in this field. In this work, we revolve around improving energy and ghostly effectiveness by using Huge MIMO methodology for LTE systems.

J. Chen et al., [2] This work stresses with a hand-off upheld tremendous multiple input multiple output (MIMO) cellular system. The exact shut structure articulations of both ghastly proficiency and energy efficiency (EE) are procured for downlink single-cell multi-customer multi-transfer tremendous MIMO transmission in the pilot-degraded everyday practice, where the amount of customers is greater than the pilot gathering length. They propose two upgrade techniques: 1-D looking and substitute improvement. About, the past achieves an unrivaled execution, while the last has a lower multifaceted nature. Amusement results endorse the practicality of the two procedures. A. He et al., [3] One of the key $5 \mathrm{G}$ circumstances is that device-to-device (D2D) and colossal multiple-input multiple-output (MIMO) will be harmonized. Nevertheless, deterrent in the uplink D2D underlaid gigantic MIMO cellular networks should be made, due to the immense cellular and D2D transmissions

P. Patcharamaneepakorn et al., [4] This work ponders summarized spatial equalization (Gen-SM) plots in multicell multiuser colossal multiple-input multiple-output (MIMO) systems as a promising high-throughput and energy-successful methodology for fifth-age (5G) remote networks.. Y. Han et al., [5] Massive multiple-input multiple-output (MIMO) systems are one of key developments for front line cellular giving high ghastly effectiveness. While the effect of most impedance and uproar vanishes as the amount of recieving wires expands, execution of huge MIMO structures is obliged by pilot contamination realized by compared pilot. Z. Tooth et al., [7] This work shows the new assessment of the uses of massive multiple-input-multiple-output (MIMO) in full-duplex (FD) cellular two-way transfer networks, and sheds gainful bits of information on the joint efforts between gigantic MIMO, and hand-off and duplex modes.

S. Akbar et al., [10] Full duplex (FD) correspondence has ascended as an appealing response for expanding the system throughput, by allowing downlink (DL) and uplink (UL) transmissions in a comparable range. B. Huang, et al., [11] In this work, we give a general framework to investigate the tradeoff between unearthly productivity and energy efficiency (EE) in gigantic multiple input multiple output (MIMO) engaged heterogeneous networks (HetNets) with remote backhaul. A. He, L. Wang et al., [13] One of key 5G circumstances is that device-to-device (D2D) and gigantic multiple-input multiple-output (MIMO) will be existed together. U. Amin et al., [14] Multiuser MIMO with tremendous gathering device displays is a promising advancement for future cellular networks as it offers higher unearthly productivity and decline in transmit control.

Emil Bjornson Et al. researched the breaking point and estimation accuracy of tremendous MIMO systems with no-impeccable handset gear .The examination relied upon another structure model that models the hardware impedance at each accepting wire by an additional substance twisting disturbance that is comparative with the sign force at this getting wire. They showed that hardware impedances make non zero estimation botch floors and constrained rooftops in both the connections of SNR. As far as possible areare under non impeccable supplies are generally come at a lot less gathering contraption than beyond what many would consider possible for immaculate types of gear which recommend a system should benefit the results.

\section{ChanNEl CAPACITY AND MIMO SySTEM}

The maximum channel capacity of a MIMO system, the channel capacity can be estimated as a function of $\mathrm{N}$ spatial streams. A basic approximation of MIMO channel capacity is a function of spatial streams, bandwidth, and signal-to-noise ratio (SNR) and is shown in the following Eq.:

$$
\text { Capacity }=N B W \log 2(1+S N R)
$$

Given the equation for MIMO channel capacity, it is possible to investigate the relationship between the number of spatial streams and the throughput of various implementations of SISO and MIMO configurations.

For the LTE downlink, a 2x2 configuration for MIMO is assumed as baseline configuration, i.e. 2 transmit antennas at the base station and 2 receive antennas at the terminal side. Configurations with 4 antennas are also being considered. Different MIMO modes are envisaged. It has to be differentiated between spatial multiplexing and transmit diversity, and it depends on the channel condition which scheme to select. 


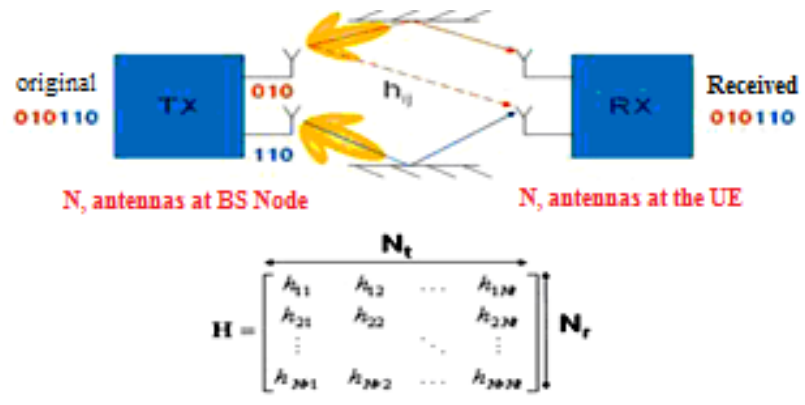

Figure 1 Down link of MIMO system

The assumptions mentioned in the section 1 will be proven to be adequate by measurements later. With these assumptions we can use this mathematical model

$$
r_{t}=H s_{t}+v_{t}
$$

Where $\mathrm{r}_{\mathrm{t}}=\left[r_{t}^{1} r_{t}^{2} \ldots r_{t}^{n_{r}}\right]^{T}$ is the received signal at time instant $\mathrm{t}$, $\mathrm{s}_{\mathrm{t}}=\left[\mathrm{S}_{t}^{1}, \mathrm{~s}_{t}^{2}, \ldots, S_{t}^{n_{T}}\right]^{T}$ is the sent signal and $\mathrm{v}_{\mathrm{t}}$ is AWGN with unit variance and uncorrelated between the $n_{r}$ receiver antennas. Receiver antenna i receive a superposition of every sent messages from transmitter $\mathrm{j}$, weighted by the channel response and some AWGN are added. The $n_{R} \times n_{T}$ transition matrix is made up of elements $h_{i, j}$ as follows

$$
H=\left(\begin{array}{ccc}
h_{1,1} & \ldots & h_{1, n_{T}} \\
\ldots & \ldots & \ldots \\
h_{n_{R}, 1} & \ldots & h_{n_{R}, n_{T}}
\end{array}\right)
$$

Where $h_{i, j}$ denotes the complex channel coefficient between the $\mathrm{j}$ :th transmit antenna and the i:th receiver antenna

\section{RESUlts AND EVALUATION OF CAPACITY}

\section{Experiment !: Channel Capacity as function of the system} size.

Comparison and validation of the calculated channel capacity of the basic standard single antennas system is given in Figure 3 and the simple model of MIMO system with $4 \times 4$ antenna size. As a function of the SNR in $\mathrm{dB}$

When comparing systems of different sizes, we need to normalize the channel matrix. The channel matrix is normalized such that, $\|H\|_{F}^{2}=n_{T} \cdot n_{R}$, where $\|\cdots\|_{F}^{2}$ represent the Frobenius norm. This normalization removes the influence of the variation in time and frequency but keeps the spatial characteristics, which is of interest here.

The channel is estimated at the receiver end with the utilization of a small well known transmitted training sequence. For a every antenna system one can apply the Shannon capacity formula as:

$$
C=\log _{2}(1+S / N)
$$

Where $\mathrm{C}$ is the channel capacity and is measured in [Bits/(sec*Hz)],

$\mathrm{B}$ is the bandwidth and

$\mathrm{S} / \mathrm{N}$ is the signal-to-noise ratio.

This channel capacity is defined as the maximum rate the channel can give with arbitrary low probability of bit errors (allowing infinite coding delay). Hence, it is an upper limit on the practical achievable bit-rate. When one uses a MIMO system one have to use a generalized version of Shannon's formula:

$$
\begin{array}{r}
C=\log _{2}\left(\operatorname{det}\left(I+\frac{\rho_{k} \cdot H H^{*}}{\sigma^{2}}\right)\right)= \\
\sum_{k=1}^{n} \log _{2}\left(1+\frac{\rho_{k}}{\sigma^{2}} \lambda_{k}\right)
\end{array}
$$

Where $\mathrm{H}$ is the transition matrix of the modeled channel and $\left(^{*}\right)$ denotes the complex conjugate transpose.

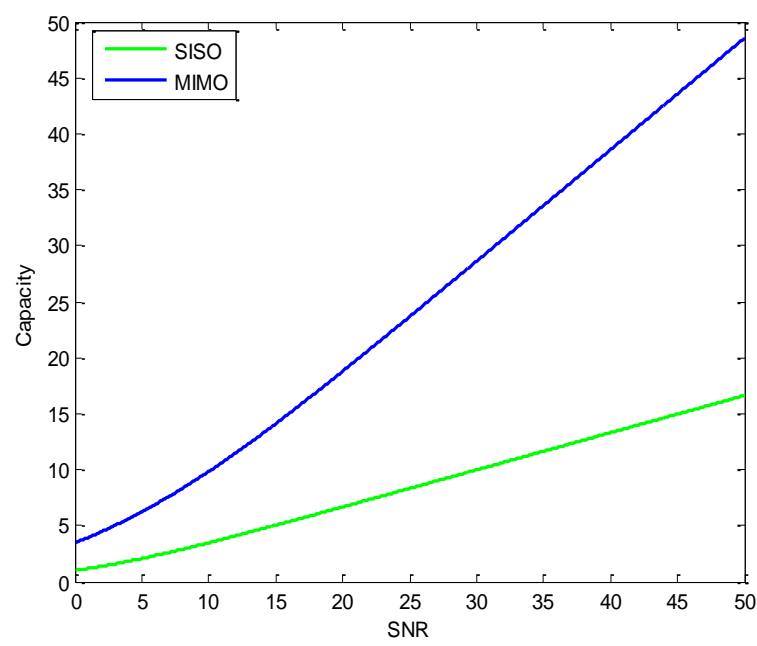

Figure 3 comparison and validation of the calculated channel capacity of the basic standard single antennas system and the simple model of MIMO system with 4 x 4 antenna size. As a function of the SNR in $\mathrm{dB}$

Where parameters are defined as

$\rho_{k}, \mathrm{k}=1,2, \ldots, \mathrm{n}$ is the transmitted energy through channel mode $\mathrm{k}$, and $\lambda_{k}$. is the power gain of the $\mathrm{k}^{\text {th }}$ node.

As a consequence of $\sigma^{2}$ as the variance of the power It can be observed from the Figure 6.3 that the result of the experiment 2 of the thesis presents the comparison and evaluation of the calculated channel capacity of the basic standard single antennas system SISO and the simple model of MIMO system with 4 x 4 antenna size as a function of the SNR in $\mathrm{dB}$.

It can be observed from the Figure. 3 that the MIMO capacity increases as the number or the size of the MIMO channel in terms of the channel states and matrix dimensions 'or antennas increases. During this experiment 2 the SNR is significantly increased from 0 to almost 50 at an interval of the 5. Thus it can be observed that at as the SNR of the system increases the channel capacity of MIMO increases exponentially. and a SNR $=50$ the channel capacity become almost triple of the SISO system.

\section{A Impact of hardware impairment on the channel capacity of MIMO}

This section considers the small amount of hardware impairment within the system. With these assumptions the channel model is reformulated mathematically as

$$
r_{t}=K^{B S} H s_{t}+K^{U E} H s+v_{t}
$$




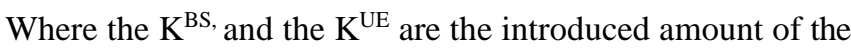
hardware impairment in the form of the Kapa values now with this model the channel capacity of the basic MIMO system is modeled as the

$$
\begin{gathered}
C_{M I M O_{i m p}}=C_{M I M O_{i m p}}+ \\
\log 2\left(1+\frac{S N R * K^{B S} * \lambda_{k}}{N t}+\frac{S N R * K^{U E} * \lambda_{k}}{N t}\right)
\end{gathered}
$$

This channel capacity is compared with the SISO and MIMO standard systems for the comparison and impact of the hardware impairment on the system performance.

It can be observed from the Figure 4 that, the capacity of the MIMO system significantly degrades under the presence of the hardware impairment in the system. The capacity reduces to 53 from the 66 for the $\mathrm{SNR}=50 \mathrm{~dB}$ with $4 \times 4$ antenna configuration as clear from the Figure 4

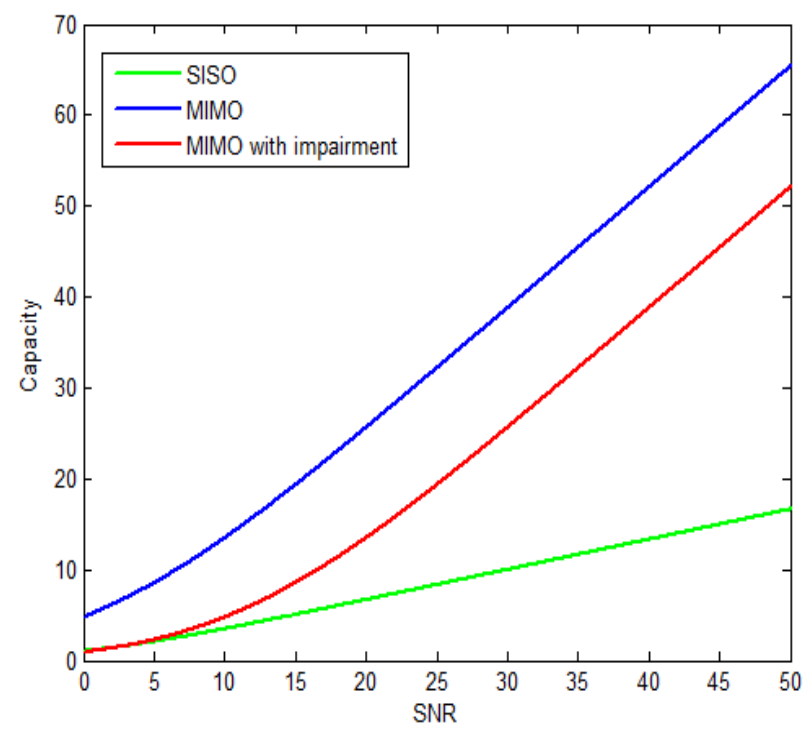

Figure 4 Impact of the hardware impairment on the performance of the channel capacity considering the

$$
K^{B S}=K^{U E}=0.05
$$

\section{CHANNEL CAPACITY OF ANTENNA CONFIGURATIONS UNDER HARDWARE IMPAIRMENT}

As another experiment the performance of the channel capacity of the various antenna configurations are compared as the function of the SNR values. It can be observed from the Figure 4 that for experiment 2 the comparison of channel capacity of various antenna configurations with the simple model of MIMO system with $2 \times 2$ sizes is presented as a function of the SNR in $\mathrm{dB}$.

It can be observed that the MIMO capacity is highs with the use of the MIMO system due to the multi path environment.

$$
\begin{gathered}
C_{\text {SISO }}(\mathrm{K})=C_{\text {SISO }}(\mathrm{K}) \\
+\log 2\left(1+\operatorname{SNR}(\mathrm{K}) * \operatorname{norm}\left(\mathrm{h}_{\text {SISO }}\right)^{2}\right) \\
C_{\text {SIMO }}(\mathrm{K})=C_{\text {SIMO }}(\mathrm{K}) \log 2(1+\operatorname{SNR}(\mathrm{K}) \\
\left.* \operatorname{norm}\left(h_{\text {SIMO }}\right)^{2}\right) ;(4)
\end{gathered}
$$

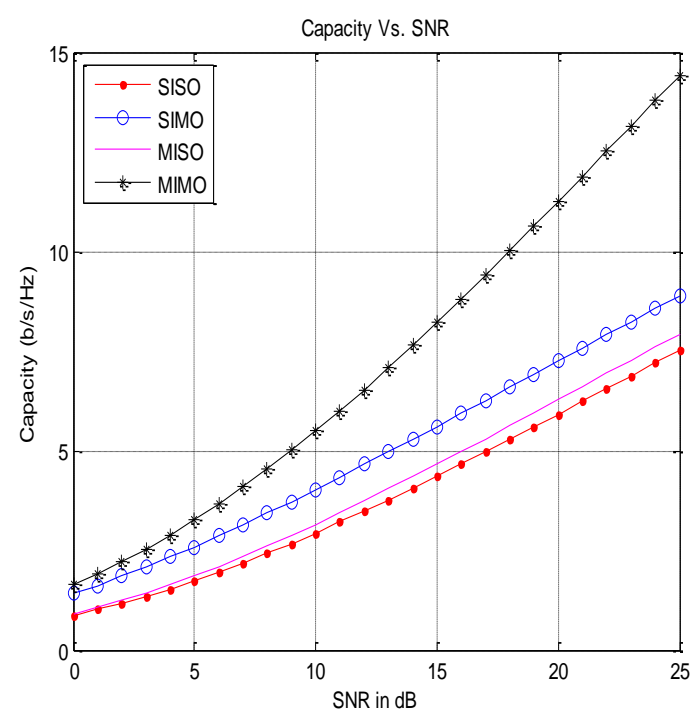

Figure 6.4 evaluation of performance of the calculated channel capacity for the various antenna configurations as SISO, SIMO, MISO and MIMO with the simple $2 \times 2$ antenna size. as a function of the SNR in $\mathrm{dB}$

$$
\begin{gathered}
C_{\text {MISO }}(\mathrm{K})=C_{M I S O}(\mathrm{~K}) \\
+\log 2\left(1+\operatorname{SNR}(\mathrm{K}) * \frac{\operatorname{norm}\left(h_{M I S O}\right)^{2}}{\mathrm{mT}}\right)
\end{gathered}
$$

$$
\begin{array}{r}
C_{M I M O}(\mathrm{~K})=C_{\text {MIMO }}(\mathrm{K}) \log 2(\operatorname{abs}(\operatorname{det}(\operatorname{eye}(\mathrm{mR}) \\
\left.\left.\left.+\operatorname{SNR}(\mathrm{K}) * h_{M I M O} * \frac{h_{M I M O}}{\mathrm{mT}}\right)\right)\right)
\end{array}
$$

Then finally these channel capacities are normalized with respect to number of Montecalo iterations (ITER) to generate final channel capacity as mentioned in the respective equations below

$$
\begin{array}{r}
C_{\text {SISO }}=C_{\text {SISO }} / \text { ITER; } \\
C_{\text {SIMO }}=C_{\text {SIMO }} \overline{\text { ITER }} ; \\
C_{\text {MISO }}=C_{\text {MISO }} / \text { ITER; } \\
C_{M I M O}=C_{\text {MIMO }} / \text { ITER } ;
\end{array}
$$

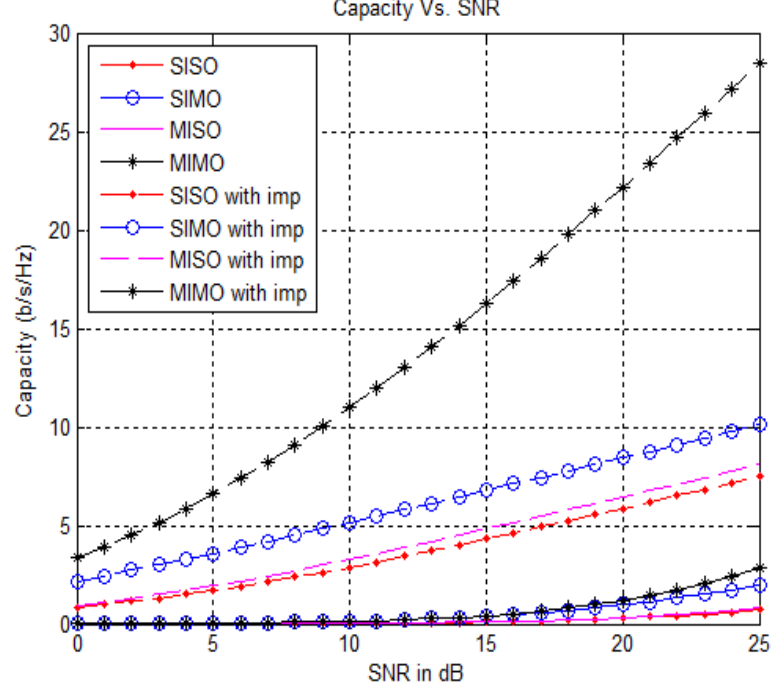

Figure 6.6 impact of the impairment on the MIMO capacity bounds 


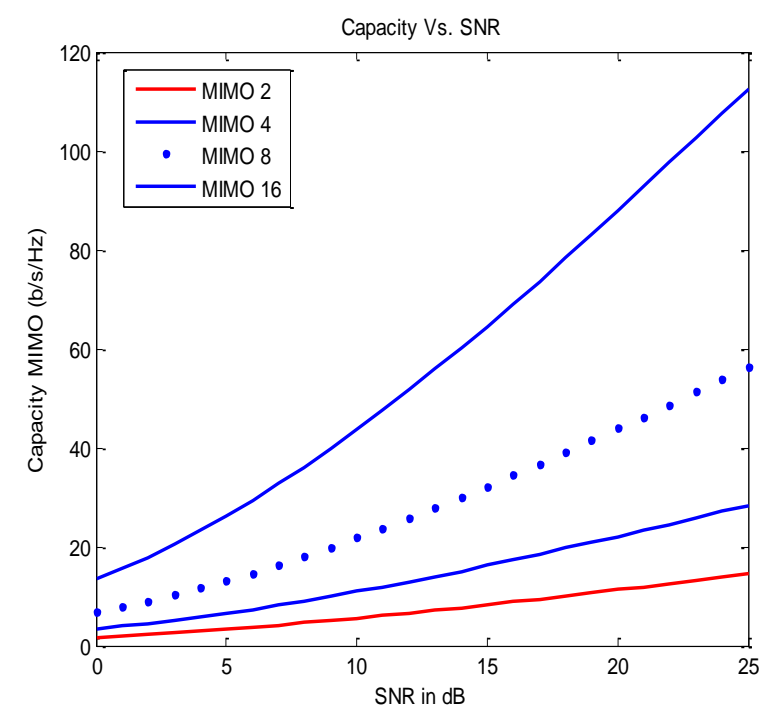

Figure 6.Results of the evaluation of performance of channel capacity with the increasing size of the MIMO system as order of the 2 with symmetric channel matrix as the function of the SNR in $\mathrm{dB}$

This Figure 5 compares the performance of the capacity under the different MIMO sizes. It can be observed from the Figure 6.5 that capacity of the transmission for the MIMO system increases significantly of the increasing size of the MIMO channel model.

\section{CONCLUSION}

In this paper the performance of the MIMO antenna configurations are tested under the presence of the hardware impairment in the system. The performance is evaluated and analysed based on the channel capacity bound of the MIMO systems. The new capacity formulations are defined for the capacity under impairments for antenna configurations. The capacity reduces to 53 from the 66 for the $\mathrm{SNR}=50 \mathrm{~dB}$ with 4 $\mathrm{x} 4$ antenna configuration. It is conclude the channel capacity of MIMO system increases with the increase in the size of the systems. calculated channel capacity of the basic standard single antennas system SISO and the simple model of MIMO system with $4 \times 4$ antenna size as a function of the SNR in $\mathrm{dB}$. But under the impairments there is significant reduction of around $1 / 3$ rd in the capacity of the channels. Thus it is proposed to improve the performance under the impairment by using the efficient channel estimation methods. /

\section{ACKNOWLEDGMENT}

The authors acknowledges to each and every person associated to this research work. And we acknowledge the all authors referred here.

\section{REFERENCES}

1. Y. Gizlenmistir, "Enormous MIMO in LTE frameworks: Vitality and otherworldly productivity," 2018 26th Sign Handling and Correspondences Applications Meeting (SIU), Izmir, 2018, pp. 1-4.

2. J. Chen, H. Chen, H. Zhang and F. Zhao, "Otherworldly Vitality Effectiveness Tradeoff in Hand-off Helped Huge MIMO Cell Systems With Pilot Pollution," in IEEE Access, vol. 4, pp. 5234-5242, 2016.

3. A. He, L. Wang, Y. Chen, K. Wong and M. Elkashlan, "Otherworldly and Vitality Productivity of Uplink D2D Underlaid Monstrous MIMO Cell Systems," in IEEE Exchanges on Interchanges, vol. 65, no. 9, pp. 3780-3793, Sept. 2017.

4. P. Patcharamaneepakorn et al., "Otherworldly, Vitality, and Monetary Proficiency of 5G Multicell Gigantic MIMO Frameworks With Summed up Spatial Balance," in IEEE Exchanges on Vehicular Innovation, vol. 65, no. 12, pp. 9715-9731, Dec. 2016.

5. Y. Han and J. Lee, "Grassmannian preparing for huge MIMO cell systems," 2016 50th Asilomar Meeting on Signs, Frameworks and PCs, Pacific Woods, CA, 2016, pp. 193-197.

6. X. Lin, R. W. Heath and J. G. Andrews, "The Interaction Between Huge MIMO and Underlaid D2D Systems administration," in IEEE Exchanges on Remote Correspondences, vol. 14, no. 6, pp. 3337-3351, June 2015.

7. Z. Tooth, W. Ni, F. Liang, P. Shao and Y. Wu, "Enormous MIMO for Full-Duplex Cell Two-Way Transfer System: A Phantom Productivity Study," in IEEE Access, vol. 5, pp. 23288-23298, 2017.

8. W. Liu, S. Jin, C. Wen, M. Matthaiou and X. You, "A Tractable Way to deal with Uplink Phantom Effectiveness of Two-Level Enormous MIMO Cell HetNets," in IEEE Interchanges Letters, vol. 20, no. 2, pp 348-351, Feb. 2016.

9. D. Verenzuela, E. Björnson and L. Sanguinetti, "Phantom and Vitality Effectiveness of Superimposed Pilots in Uplink Enormous MIMO," in IEEE Exchanges on Remote Interchanges, vol. 17, no. 11, pp. 7099-7115, Nov. 2018.

10. S. Akbar, Y. Deng, A. Nallanathan, M. Elkashlan and G. K. Karagiannidis, "Huge Multiuser MIMO in Heterogeneous Cell Systems With Full Duplex Little Cells," in IEEE Exchanges on Correspondences, vol. 65, no. 11, pp. 4704-4719, Nov. 2017.

11. B. Huang, A. Guo and A. Bo, "Vitality and Phantom Effective Tradeoff for Huge MIMO Empowered Heterogenous Systems with Remote Backhaul," 2018 IEEE eighteenth Universal Gathering on Correspondence Innovation (ICCT), Chongqing, 2018, pp. 832-837

12. S. Mandal and S. Gauni, "Vitality effectiveness of single cell and multi cell Huge MIMO framework MMSE estimation," 2017 Worldwide Meeting on Nextgen Electronic Advancements: Silicon to Programming (ICNETS2), Chennai, 2017, pp. 66-70.

13. A. He, L. Wang, Y. Chen, K. Wong and M. Elkashlan, "SE and EE of Uplink D2D Underlaid Enormous MIMO Cell Systems with Force Control," 2017 IEEE Remote Interchanges and Systems administration Meeting (WCNC), San Francisco, CA, 2017, pp. 1-6.

14. U. Amin, S. Anwar, W. Khan and S. I. Khan, "Vitality productivity investigation of single-cell enormous MIMO under pilot sullying," 2016 thirteenth Global Bhurban Gathering on Applied Sciences and Innovation (IBCAST), Islamabad, 2016, pp. 704-709.

15. Emil Bjornson, Jakob Hoydis, Marios Kountouris,, Merouane Debbah, Enormous MIMO Frameworks with Non-Perfect Equipment: Vitality Proficiency, Estimation, and Limit Points of confinement. IEEE trisection 2014.2014.

\section{AUTHORS PROFILE}

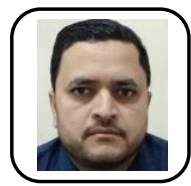

Prof Mohd Amzad, has joined Sagar Group in July 2013 .He is pursuing $\mathrm{PhD}$ in Communication \& Antenna design from AISECT university Bhopal. He has 12 years of experience in teaching and year experience in industry. He has conducted several skill development program and workshops for students

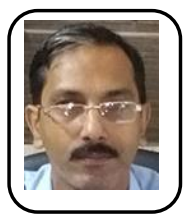

Prof. Paresh Rawat, is doctorate in Electronics and Communication Engineering and has rich experience of 15 years in academics. He has received $\mathrm{PhD}$ in Electronics \& Communication from Maulana Azad National Institute of Technology (MANIT) in 2014. He is awarded M. Tech in Digital Communication in 2007 from MANIT, Bhopal. He has received Bhaskar Teachers Facilitation Award 2016, and has been awarded as Best faculty in Electronics and Communication Branch in CII SISTEC Tech Guru Award 2017 and best faculty award in Electronics by Truba Group of Institute in 2013. He is certified faculty by IBM. 